OPEN

SUBJECT AREAS:

RADIOTHERAPY

CELL BIOLOGY

PHYSICS

DNA

Received

10 January 2013

Accepted

12 April 2013

Published

3 May 2013

Correspondence and requests for materials should be addressed to J.N.K. (j.kavanagh@ qub.ac.uk)

\section{Antiproton induced DNA damage: proton like in flight, carbon-ion like near} rest

\author{
J. N. Kavanagh', F. J. Currell 1,2, D. J. Timson ${ }^{3}$, K. I. Savage' , D. J. Richard ${ }^{4}$, S. J. McMahon' ', O. Hartley ${ }^{5}$, \\ G. A. P. Cirrone ${ }^{6}$, F. Romano ${ }^{6}$, K. M. Prise' ${ }^{1}$, N. Bassler ${ }^{7,8}$, M. H. Holzscheiter ${ }^{9}$ \& G. Schettino'
}

${ }^{1}$ Centre for Cancer Research and Cell Biology, Queen's University Belfast, Belfast, UK, ${ }^{2}$ Centre for Plasma Physics, School of Mathematics and Physics, Queen's University Belfast, Belfast, UK, ${ }^{3}$ School of Biological Sciences, Queen's University Belfast, Belfast, UK, ${ }^{4}$ Institute of Health and Biomedical Innovation, Queensland University of Technology, Brisbane, Australia, ${ }^{5}$ Department of Structural Biology and Bioinformatics, University of Geneva, Geneva, Switzerland, ${ }^{6}$ INFN-LNS, Catania, Italy, ${ }^{7}$ Department of Experimental Clinical Oncology, Aarhus University Hospital, Aarhus, Denmark, ${ }^{8}$ Department of Physics and Astronomy, Aarhus University, Aarhus, Denmark, ${ }^{9}$ Department of Physics and Astronomy, University of New Mexico, Albuquerque, NM, USA.

Biological validation of new radiotherapy modalities is essential to understand their therapeutic potential. Antiprotons have been proposed for cancer therapy due to enhanced dose deposition provided by antiproton-nucleon annihilation. We assessed cellular DNA damage and relative biological effectiveness (RBE) of a clinically relevant antiproton beam. Despite a modest LET $(\sim 19 \mathrm{keV} / \mu \mathrm{m})$, antiproton spread out Bragg peak (SOBP) irradiation caused significant residual $\gamma$ - $\mathrm{H} 2 \mathrm{AX}$ foci compared to $\mathrm{X}$-ray, proton and antiproton plateau irradiation. $\mathrm{RBE}$ of $\sim 1.48$ in the SOBP and $\sim 1$ in the plateau were measured and used for a qualitative effective dose curve comparison with proton and carbon-ions. Foci in the antiproton SOBP were larger and more structured compared to X-rays, protons and carbon-ions. This is likely due to overlapping particle tracks near the annihilation vertex, creating spatially correlated DNA lesions. No biological effects were observed at $28-42 \mathrm{~mm}$ away from the primary beam suggesting minimal risk from long-range secondary particles.

$\mathrm{t}$ is commonly acknowledged that a significant improvement in cancer radiotherapy requires both a better control of energy deposition and a greater knowledge of the biological response of the irradiated tissues. Charged particles (Hadron-therapy) have been suggested for cancer treatment, and are currently being used with impressive results in clinics worldwide, because they exhibit an inverse depth-dose profile with most of their energy deposited at the end of the particle track in what is known as the 'Bragg peak'. Compared to conventional Mega Voltage (MV) X-ray radiotherapy, hadron-therapy offers a clear dosimetric advantage as it allows a reduction of the integral dose to healthy tissue by a factor of $2-3^{1}$. Moreover, unlike X-rays, the biological response of samples exposed to charged particle beams changes as particles slow down as they penetrate the sample. This could be further exploited for radiotherapy purposes as enhanced biological effectiveness is expected in the final part of the particle track due to the increasing Linear Energy Transfer (LET). Protons are generally considered to have a similar biological effectiveness to photons (Relative Biological Effectiveness, $\mathrm{RBE}=\sim 1.1$ ). Therefore the main advantage of using protons is the possibility of delivering a lethal tumour dose with minimal dose to surrounding healthy tissue, thus reducing the risk of adverse effects. Consequently, protons are prescribed for treatment of tumours near high-risk tissues such as the spinal cord and are predicted to have the best advantage for treatment of rare pediatric cancers. In those cases the ability to minimise healthy tissue damage and dose to developing organs is $\mathrm{critical}^{2,3}$.

In the case of heavier ion beams such as carbon ions an additional advantage is provided by the increased ionization density of the final part of the ion track. The subsequent damage to critical cellular targets, primarily DNA, is therefore more challenging to repair and results in higher biological effectiveness. The LET increases significantly as carbon ions slow down, making these ions 2-5 times more effective per unit dose absorbed compared to photons (and protons) ${ }^{5-7}$. In addition, high-LET radiation has been demonstrated in numerous in vitro experiments to be more effective than low-LET-radiation for the treatment of radiation resistant and hypoxic tumours ${ }^{8}$. Unfortunately, heavy ions also have a higher RBE in the entrance channel compared to X-rays and low LET particles thereby presenting an increased damage risk for healthy tissues in the entrance channel. 
RBE range for cell killing in vitro in the entrance channel of 100$270 \mathrm{MeV} / \mathrm{u}$ carbon ions is 1 to $1.75^{5,7,9,10}$. However, since the most substantial increase in RBE is found at the distal end of the particle track, i.e. within the tumour, this leads to a net therapeutic advantage, which forms the radiobiological rationale for carbon-ion therapy.

Like proton and carbon ion beams, antiprotons display a distinct Bragg peak ${ }^{11-14}$. Given that they have an identical mass and equal, but opposite, charge to protons, antiprotons interact with matter, through Coulomb interactions; in the same way as protons do and are therefore characterized by a low LET proximal to the Bragg peak, i.e. in the therapeutic entrance channel. However, the ratio of physical doses deposited in the Bragg peak versus the plateau of an antiproton beam is approximately twice that of the equivalent proton beam. This is due to antiproton-nucleon annihilation in the target matter as the antiprotons come to rest. Of the resulting $\sim 2 \mathrm{GeV}$ released by the annihilation event, approximately $30 \mathrm{MeV}$ is deposited near the point of annihilation by high LET nuclear fragments ${ }^{15}$. Augmentation of LET by these nuclear fragments has been suggested to increase the biological effectiveness at the antiproton Bragg peak (as for carbon ions). Conversely, antiprotons can be expected to have similar properties to protons in the plateau with a modest, if any, increase in the $\mathrm{RBE}^{16,17}$ as the particle slows down. The remainder of the $2 \mathrm{GeV}$ produced by each antiproton annihilation radiates away from the annihilation point in the form of pions and gamma rays. Recent proof-of-principal experiments suggest that these pions may be used for real-time imaging of antiproton annihilation events in tissues providing "live" dose monitoring and targeting options. These studies indicate that dose verification to $\sim 1 \mathrm{~mm}$ accuracy would be possible within a therapeutic dose range ${ }^{18,19}$. However, concerns about this long range secondary radiation have been raised as it would inevitably decrease the resolution of the dose deposition profiles which is one of the main advantages of ion beam therapy ${ }^{20-22}$. It is however, recognized that the resolution of the dose profiles will have to be assessed for the biological effective dose, including RBE profiles, requiring detailed investigations of the biological consequences of antiproton exposures.

Only then can we precisely assess the risks and potentials of antimatter beams. To this purpose, we have studied the response of normal human fibroblast cells to antiproton exposure along the particle path and in its immediate surroundings. DNA damage and cell killing induced by the products of the antiproton-annihilation are compared with those caused by other ion beams, photons and out-of field effects in cells not traversed by the primary antiproton beam but exposed mainly to long range secondary particles.

\section{Results}

Antiproton annihilation causes spatially correlated DNA damage. In order to assess if antiprotons induce DNA damage of different quantity and quality to other radiation modalities, DNA double strand breaks (DSBs) were analyzed in irradiated cell nuclei at 1 and $26 \mathrm{~h}$ post irradiation. DNA DSBs were visualized by immunostaining for phospho-serine 139 histone 2AX $(\gamma-\mathrm{H} 2 \mathrm{AX})$ (Fig. 1.a), a marker of DNA DSBs ${ }^{23}$. Fluorescence imaging of $\gamma$ $\mathrm{H} 2 \mathrm{AX}$ foci indicated that antiproton SOBP irradiated nuclei contained foci that were larger than X-ray, proton, or carbon-ion irradiated samples at $1 \mathrm{~h}$ (full width at half maximum fluorescence a

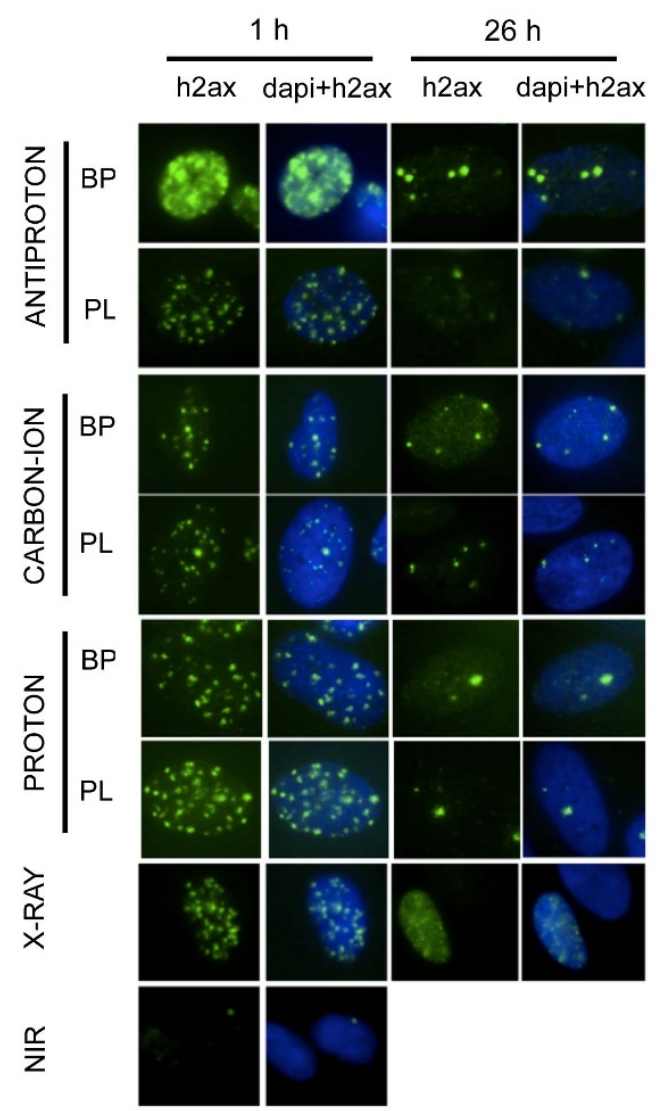

b

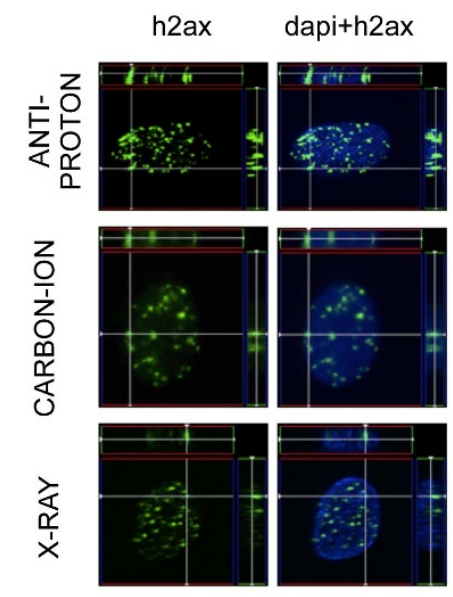

C

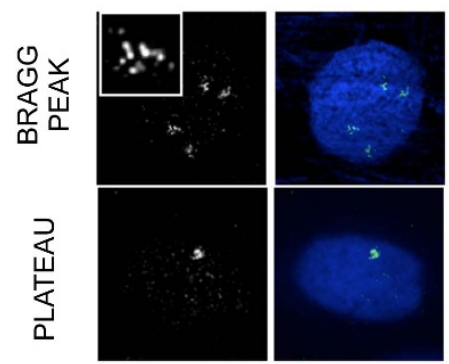

Figure $1 \mid$ Initial and residual $\gamma$-H2AX foci were observed in charged particle irradiated fibroblasts. (a) $\gamma$-H2AX foci (green) were imaged at 1 and $26 \mathrm{~h}$ after irradiation with antiprotons (top), carbon ions, protons or $225 \mathrm{kVp} \mathrm{X-rays.} \mathrm{(b)} \mathrm{'Cut-view'} \mathrm{images} \mathrm{taken} \mathrm{from} \mathrm{compiled} \mathrm{Z-stack} \mathrm{images} \mathrm{through}$ irradiated cells show stacks of foci along antiproton paths in SOBP irradiated cells that were fixed $1 \mathrm{~h}$ after irradiation. (c) Restoration deconvolution was applied to images of cell nuclei from antiproton-irradiated fibroblasts. Antiproton SOBP irradiated nuclei (top) contained large clustered foci that were smaller and less frequent in antiproton plateau irradiated fibroblasts (bottom). 
intensity $(\mathrm{FWHM})=1.58 \pm 0.13 \mu \mathrm{m}$ for antiproton SOBP against $1.26 \pm 0.07 \mu \mathrm{m}$ for carbon-ion SOBP and $0.79 \pm 0.08 \mu \mathrm{m}$ for protons $)$ and significantly larger $(\mathrm{P}<0.0001$ and $\mathrm{P}=0.0005$ respectively) at $26 \mathrm{~h}(\mathrm{FWHM}=1.65 \pm 0.12 \mu \mathrm{m}$ for antiprotons against $0.95 \pm 0.07 \mu \mathrm{m}$ for the carbon SOBP and $1.13 \pm 0.07 \mu \mathrm{m}$ for protons) post-irradiation. Results of high-resolution imaging combined with restoration-deconvolution indicated that the large $\gamma-\mathrm{H} 2 \mathrm{AX}$ foci observed in the nuclei of antiproton Bragg peak irradiated cells consisted of multiple micro-foci units (Fig. 1.c). The larger foci produced by the antiproton annihilation events appear therefore to be clusters of individual but spatially and temporally correlated DNA lesions as characteristic of high LET radiation. Imaging nuclei through fine Z-stacks also revealed that both antiproton and carbon-ion SOBPs produce elongated foci, which extend along the particle track in the direction of the particle beams. Although it was not possible to distinguish individual foci along the particle track, this is clearly the result of a series of DNA DSBs caused by the high ionization density near the Bragg peaks. This effect was not observed in samples exposed to Xray, proton and antiproton in the plateau where all foci appear as isolated small units (Fig. 1.b).

As in the case of any particle exposures, at microscopic level there will be dose inhomogeneity with a high amount of energy deposited along the particle track and less by the secondary electrons. This is accentuated in the case of heavy ions such as carbon and similar situations are expected at the site of annihilation. As the annihilation occurs predominantly at the end of the antiproton range, the frequency of annihilation per cell depends on the position of the cell within the SOBP and weighted energy spread. For the experiment reported, in the middle of the SOBP (average LET $=19 \mathrm{keV} / \mu \mathrm{m})$, a cell will experience $\sim 50$ antiproton traversals per Gy. Based on the fact that 7 different beam energies (120-126 MeV, step $\sim 1 \mathrm{MeV})$ are used to modulate the SOBP, $\sim 10 \%$ of these antiprotons (i.e. $\sim 5$ antiproton annihilation/cell per Gy) will annihilate in cells around the centre of the SOBP.

Foci size and repair kinetics. Quantification of DNA damage foci at $1 \mathrm{~h}$ post-irradiation showed that all radiation modalities investigated had a linear dose-response relationship within the LET range considered (4-150 keV/ $\mu \mathrm{m})$ (see SI Fig. S1).

At $1 \mathrm{~h}$ after irradiation there is no statistically significant change in the mean number of foci/Gy/cell as a function of LET (Fig. 2.a). However, because repair will have begun minutes after the irradiation and the repair dynamics also depends on the quality of damage, it cannot be determined from these experiments if the total initial number of DBSs was different. The number of particle traversals per cell nucleus can be estimated by the following Equation (1).

$$
\mathrm{D}(\mathrm{Gy})=\mathrm{LET}(\mathrm{keV} / \mu \mathrm{m}) \times \Phi\left(\text { particle } / \mathrm{cm}^{2}\right) \times 1.610^{-9}
$$

However, the correlation between nuclear traversals and focus formation strongly depends on the LET, as at low LET not all particle traversals would result in a focus, while at higher LET multiple foci could overlap. The kinetics of DBS repair over $26 \mathrm{~h}$ was therefore used as a measure of the ability of antiprotons to induce challenging repair substrates. At $26 \mathrm{~h}$ after irradiation, only antiproton SOBP and carbon-ion SOBP treated samples displayed significant residual damage compared to X-ray treated and control cells (Fig. 2.a). The frequency of micro-foci (Fig. 1c) was analysed qualitatively and is not included in the mean foci/Gy/cell. To test if there was a correlation of foci size with frequency of residual foci the FWHM was measured in foci from all sample sets. The FWHM of the foci was largest for antiproton SOBP treated cell nuclei at early and late times after irradiation (Fig. 2.b) while for the other radiation modalities, only the foci generated by carbon ions at the SOBP showed morphological differences at $1 \mathrm{~h}$ post irradiation. This supports the hypothesis that larger foci (or groups of foci) represent DNA lesions that may be more challenging substrates for the cellular DNA repair machinery Indeed, analysis of DNA repair kinetics in these cells (Fig. 2.c) indicated slower rates of DNA repair for antiproton SOBP exposed cells as well as for carbon ion irradiated samples (both at the proximal and distal edges of the SOBP) compared to proton and X-ray irradiated cells.

RBE in the Antiproton Bragg peak. In order to determine cellular effects of direct clinical relevance, the particle beams investigated were modulated to produce Spread-Out Bragg Peaks (SOBP) to mimic clinically relevant situations for moderate sized tumours. The physical parameters of the employed beams and respective SOBPs are found in Table 1. LET values have been calculated using Monte Carlo simulation of the experimental beam set up with Fluka for the antiproton beam and Geant 4 for the proton and carbon-ion beams. The ability of an antiproton at the midSOBP position to cause a reduction in clonogenic survival was compared to proton and carbon-ion SOBPs and $225 \mathrm{kVp}$ X-rays using the same experimental procedure. In our experiments the biological effectiveness of the antiproton SOBP was intermediate between that of carbon-ions and protons, conversely the biological effectiveness of the antiproton plateau was similar to that of X-rays. Fitting parameters for measured survival curves and calculated RBE values are found in Table 1 . The RBE at $10 \%$ survival following exposure the mid-SOBP for $126 \mathrm{MeV}$ antiproton beam was $1.48 \pm$ 0.17 , while in the plateau cell killing was comparable to that of $225 \mathrm{kVp}$ X-rays (Fig. 3.a). The RBE at 10\% survival for the proton beam was $\sim 1.2$ both proximal to, and in the middle of the SOBP (Fig. 3.b). Finally, the RBE at $10 \%$ survival for the carbon-ion beam was $2.03 \pm 0.06$ and $3.88 \pm 0.06$ respectively, for the proximal and distal edges of the SOBP (Fig. 3.c).

Long-range annihilation particles have an RBE similar to X-rays. Interactions of long-range annihilation products such as pions, neutrons and gamma rays with cells outside the primary target are of critical importance as they could alter the highly conformal dose profile field delivered with an antiproton beam and produce a wide spread low dose distribution with potential

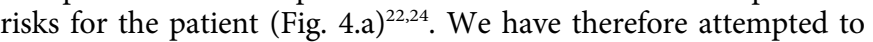
assess the biological effectiveness of long-range particles in cells adjacent to our target in the antiproton SOBP region. DNA damage foci and cell survival were assessed at different distances from the main beam in order to compare the effects with the "in beam" responses.

No statistically significant increase in $\gamma-\mathrm{H} 2 \mathrm{AX}$ foci compared to control samples (background of $1.6 \pm 0.08 \mathrm{foci} / \mathrm{cell}$ ) was detected at $1 \mathrm{~h}$ after irradiation in cells outside the primary beam except for cells located $28 \mathrm{~mm}$ from the centre of the beam when the highest dose (5.56 Gy) was delivered to the target (Fig. 4.b). In all cases, this is equal or less than that expected on the basis of the estimated dose to samples outside the primary beam. The dose at $28 \mathrm{~mm}$ from the beam centre was estimated to be less than $10 \%$ of that received by directly exposed samples for all doses. A qualitative example of the lateral dose distribution is given in SI Fig. S2, antiproton beam dosimetry has been described in detail by Bassler et al..$^{16,14,13}$ ).

The impact of secondary particles on cell survival was also investigated. In this case annihilation secondary particles seem to have a similar biological effectiveness to X-rays. Although the data seem to suggest a decrease in the effectiveness per unit dose, this is not statistically significant. Notably, any cell killing was only observed when the $>5$ Gy was delivered to samples at the beam centre (Fig. 4.c).

These experiments suggest that effective dose to samples outside of the main antiproton beam falls off very quickly and has a very low RBE for both cell killing and DNA DSB induction. 
a

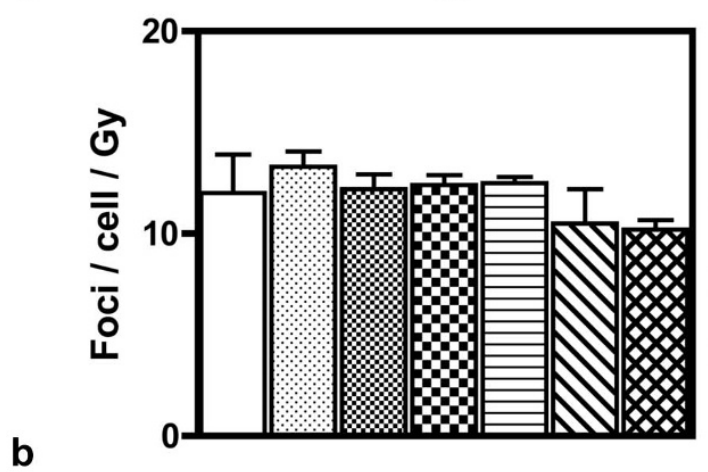

b

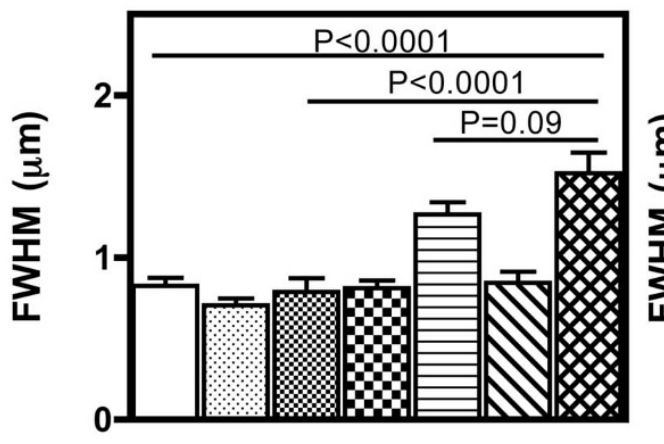

$26 \mathrm{~h}$
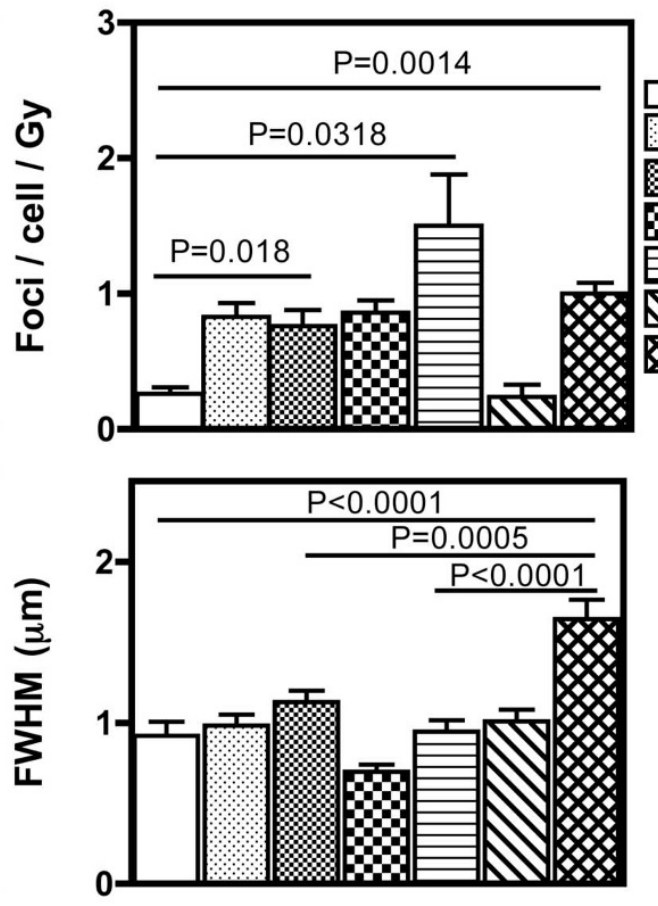

C

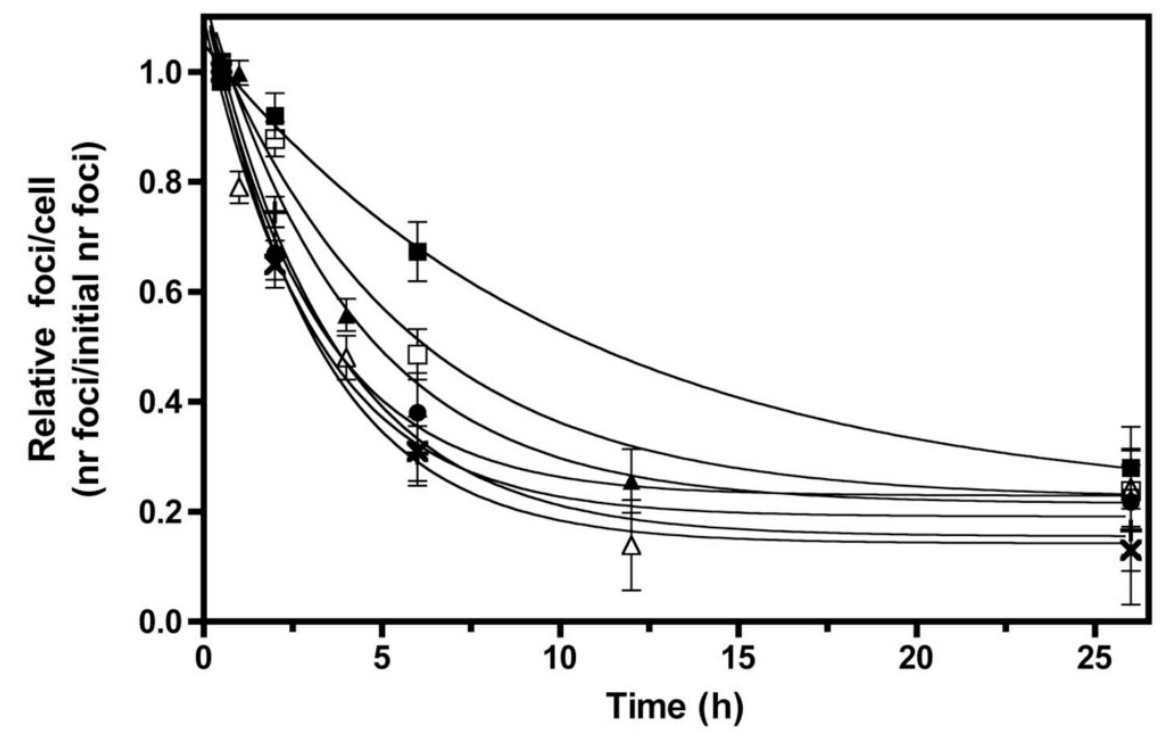

- $225 \mathrm{kVp}$ X-rays

$\times \mathrm{H}^{+} \sim 2 \mathrm{keV} / \mu \mathrm{m}(2 \mathrm{~mm})$

$+\mathrm{H}^{+} \sim 6 \mathrm{keV} / \mu \mathrm{m}(18 \mathrm{~mm})$

$\mathrm{C}^{12} \sim 70 \mathrm{keV} / \mathrm{\mu m}$

- $\mathrm{C}^{12} \sim 150 \mathrm{keV} / \mathrm{\mu m}$

$\Delta \mathrm{H}^{-} \sim 4 \mathrm{keV} / \mu \mathrm{m}$

- $\mathrm{H}^{-} \sim 19 \mathrm{keV} / \mathrm{um}$

Figure $2 \mid$ Kinetics of antiproton induced $\gamma$-H2AX foci. (a) The mean number of foci/cell/Gy was quantified in cells exposed to X-rays, $126 \mathrm{MeV}$ antiprotons (in plateau or mid-SOBP), $62 \mathrm{MeV}$ protons (proximal to or in the middle of the SOBP) and $62 \mathrm{MeV} / \mathrm{u}$ carbon ions (70 or $150 \mathrm{keV} / \mu \mathrm{m}$. The data are presented as the mean of three independent experiments, normalized to sham irradiated controls. Error bars represent the standard error. (b) The full-width at half maximum (FWHM, $\mu \mathrm{m}$ ) were calculated for foci in 30 nuclei of each group. (c) The foci resolution kinetics was plotted as a measure of DNA damage repair. Foci were scored in $>100$ nuclei per slide. The data presented are the mean and standard error.

\section{Discussion}

The purpose of the present work is not to discuss the technical and economic feasibility of antiprotons for cancer radiation treatment but to provide experimental evidence of the relative biological effectiveness of antiproton exposure to help assess its limits and potential. Therefore, this study aimed to investigate DNA damage induction/ repair and the resulting cellular effectiveness along the path of a clinically relevant antiproton beam and in its surroundings and compare this to other clinically relevant radiation modalities currently used for patient treatment. As DNA damage induction and repair and biological effectiveness are critical parameters for the successful utilization of particle beams in radiotherapy, data reported in this study provides experimental biological evidence upon which the potential of antiproton beams can be assessed.

DNA damage and survival data indicate that anti-proton annihilation events not only boost the dose in the SOBP but also enhance the biological effectiveness of the final part of the antiproton path, without significantly affecting the response in the plateau or in regions outside the beam trajectory. Our data indicate a correlated pattern of DNA damage induced by antiprotons in the SOBP. DNA damage foci in the SOBP appeared larger compared to those produced in the plateau or by the other radiation beams employed (i.e. $225 \mathrm{kVp}$ X-rays, protons and carbon ions, Fig. 1.a). Intriguingly, while a trend for larger foci with very high LET particles (up to 
Table 1 | Description of experimental radiation sources used and spread out Bragg peaks employed for antiproton, proton and carbon ion beams and linear quadratic fitting parameters and RBE for each survival curve

\begin{tabular}{|c|c|c|c|c|c|c|c|c|}
\hline & Incident energy & $\begin{array}{c}\text { Range in } \\
\text { water }(\mathrm{mm})\end{array}$ & $\begin{array}{l}\text { SOBP mid-point } \pm \\
\text { half SOBP depth } \\
(\mathrm{mm})\end{array}$ & $\begin{array}{c}\text { Sample } \\
\text { position (depth } \\
\text { in water }(\mathrm{mm}) \text { ) }\end{array}$ & $\begin{array}{l}\text { Dose av. LET } \\
(\mathrm{keV} / \mu \mathrm{m})\end{array}$ & $\alpha \pm$ s.e. $\left(G y^{-1}\right)$ & $\beta \pm$ s.e. $\left(G y^{-2}\right)$ & $\begin{array}{c}\text { RBE (SF0.1) } \\
\text { ( } \pm \text { s.e.) }\end{array}$ \\
\hline X-ray * & $225 \mathrm{kVp}$ & $n / a$ & $\mathrm{n} / \mathrm{a}$ & $n / a$ & $\sim 2$ & $0.61 \pm 0.1$ & $0.02 \pm 0.05$ & 1 \\
\hline Proton & $62 \mathrm{MeV}$ & 29.1 & $18.6 \pm 10.2$ & 2 & $\sim 2$ & $0.57 \pm 0.06$ & $0.047 \pm 0.01$ & $1.22 \pm 0.07$ \\
\hline Proton & $62 \mathrm{MeV}$ & 29.1 & $18.6 \pm 10.2$ & 18 & $\sim 6$ & $0.53 \pm 0.05$ & $0.035 \pm 0.009$ & $1.2 \pm 0.06$ \\
\hline Carbon ion & $62 \mathrm{MeV} / \mathrm{u}$ & 10.8 & $6.8 \pm 0.7$ & 3 & $\sim 70$ & $0.96 \pm 0.08$ & $0.094 \pm 0.03$ & $2.03 \pm 0.06$ \\
\hline Antiproton & $126 \mathrm{MeV}$ & 116.2 & $100 \pm 5$ & 100 & $\sim 19$ & $1.05 \pm 0.07$ & 0 & $1.48 \pm 0.17$ \\
\hline
\end{tabular}

a

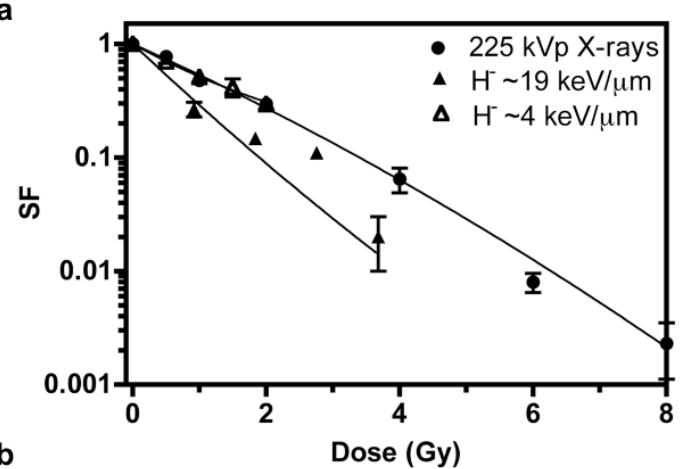

b

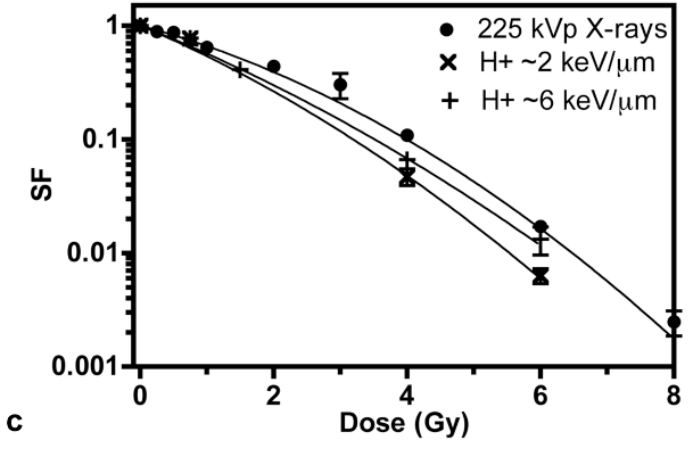

c

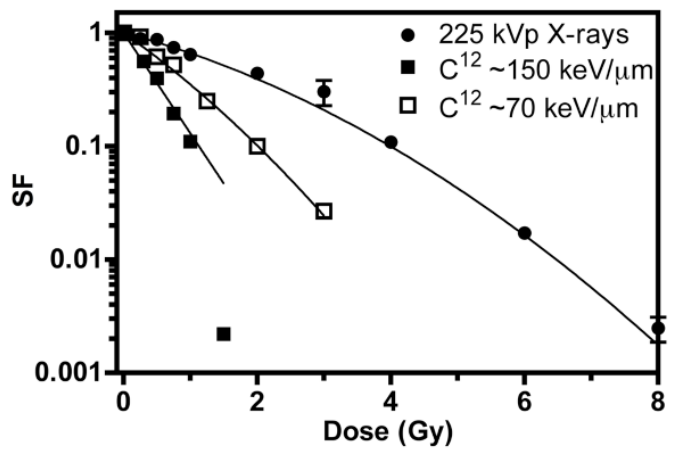

Figure 3 Cell survival and RBE estimations. Survival of AG01522 cells as a function of dose when the cells were irradiated with (a) $126 \mathrm{MeV}$ antiprotons, (b) $62 \mathrm{MeV}$ protons and (c) $62 \mathrm{MeV} / \mathrm{u}$ carbon ions. Error bars indicate the standard error of measurements from at least two independent experiments. X-ray, proton and carbon ion measurements were all performed three times. Antiproton measurements were performed three times for all doses except the highest two, which were performed twice. Survival data are fit to a weighted linear quadratic model.
$1000 \mathrm{~s} \mathrm{keV} / \mu \mathrm{m}$ ) has been reported previously by other investigators, our data indicate that foci caused by antiprotons were significantly larger than those induced by carbon ions despite their modest average LET $(19 \mathrm{keV} / \mu \mathrm{m}$ for antiprotons in the SOBP compared to 70 and $150 \mathrm{keV} / \mu \mathrm{m}$ for carbon ions used in this study) (Fig. 2.b) ${ }^{25}$. Therefore, average LET may not be the best parameter to evaluate biological effectiveness, especially in mixed radiation field situations such as those occurring following the fragmentation of heavy ions or annihilation events. By applying high resolution imaging in combination with restoration deconvolution we have shown that antiproton SOBP induced foci are morphologically distinct from those produced by X-rays, protons and carbon ions and contain 2-7 structures that closely resemble the individual foci of low LET radiation (Fig. 1.c). As is the case of any ion beam, the majority of the ionizations resulting from antiproton exposures $(\sim 80 \%)$ are still caused by delta electrons. However, the clusters of breaks identified are likely to be caused by low energy secondary particles generated by the annihilation events. Monte Carlo simulations of annihilation particles indicate that DSBs near the annihilation vertex are mostly resulting from particles with $\mathrm{Z}=1$ and helium ions. These particles are low energy $(0.16 \mathrm{MeV})$ protons with LET $\sim 71 \mathrm{keV} / \mu \mathrm{m}$ and alpha particles $(1.7 \mathrm{MeV} / \mu \mathrm{m})$ with $\mathrm{LET} \sim 72 \mathrm{keV} / \mu \mathrm{m}$. These two main responsible particles have a range of 2 and $60 \mu \mathrm{m}$, respectively (see Table 2, the full range of particles is shown in supplementary Fig. 3). Although other heavier particles are produced, their relative contribution is negligible due to the frequency of their production and their energy and range being higher. The range of the proposed DSB inducing particles is such that it may explain the large foci observed and resolution of clusters of micro-foci that indicate the presence of distinct DSBs that are spatially correlated. Such particles are generated with spatial and temporal coincidence that confers a unique pattern to the DNA damage they produce. Complex DNA lesions have been described extensively in the literature as breaks of the DNA structure at the nanometre scale ( $\sim 10$ base pairs) that can be essentially considered as single lesions with implication for the repair mechanisms due to the proximity of the damage sites. The DNA lesions described here appear to be of a different nature. In the case of heavy ions clustered DNA damage is created by high ionization density along the primary particle path ${ }^{26-31}$. High LET radiation can also cause damage over larger distances (i.e. nucleosome and chromatin fibre) and still result in single foci, although having a more relevant impact on the cellular outcome. For antiprotons, we have shown that despite a modest average LET, correlated DNA damage is produced in the Bragg peak region and propose that the large foci in antiproton SOBP irradiated cell nuclei are a product of the mixed particle field that is created around antiproton annihilations, and is not only due to delta electrons. Kreipl and colleagues have predicted from Monte Carlo simulations that an increased biological effectiveness in cases where spatially and temporally overlapping particle 
a

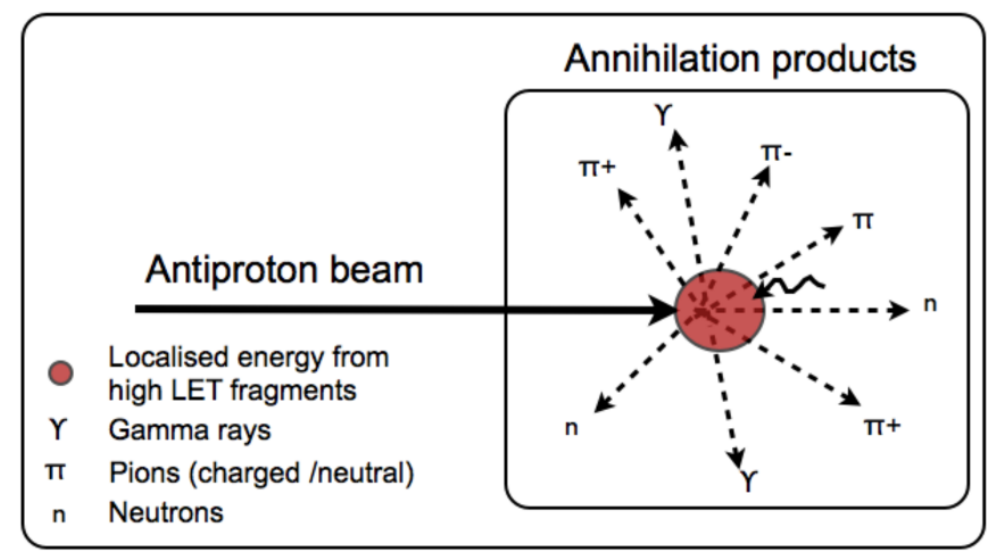

b
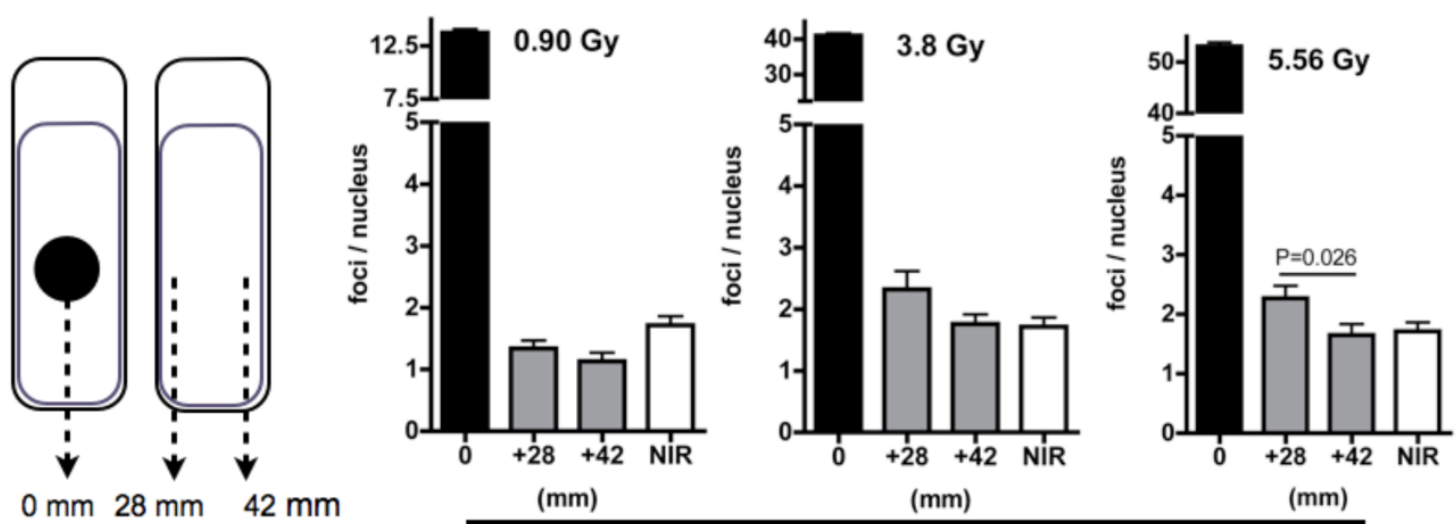

Distance from beam centre

C
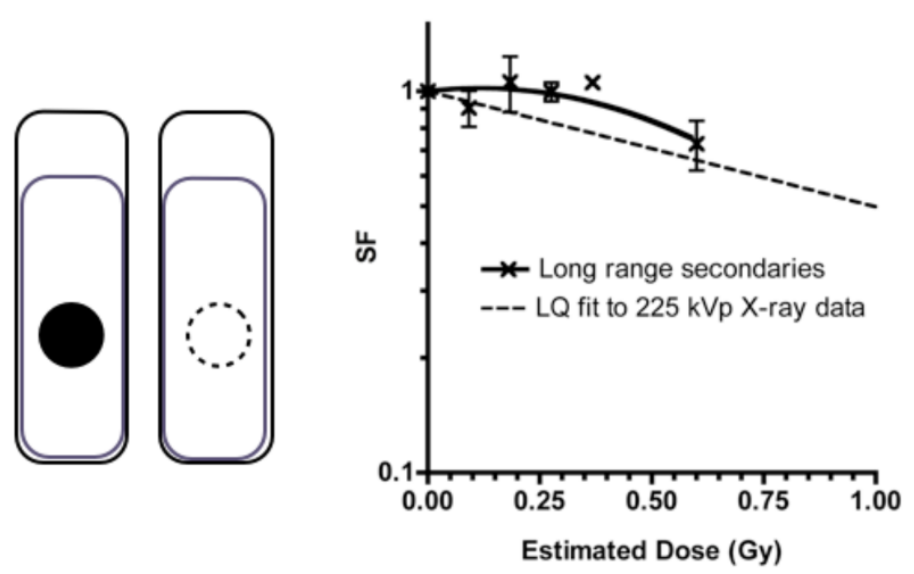

Figure $4 \mid$ Long-range annihilation particles have RBE similar to X-rays. (a) Antiproton-nucleon annihilation produces a number of long range particles such gamma rays, neutrons and positive, negative and neutral pions. (b) $\gamma$-H2AX foci were scored in cells positioned directly in the beam at the mid-point of the SOBP (solid black circle) and in an adjacent flask at either 28 or $42 \mathrm{~mm}$ from the centre of the beam $(0 \mathrm{~mm})$. A significant increase in DNA damage was only observed at $1 \mathrm{~h}$ after exposure when the in-beam target received $5.56 \mathrm{~Gy}$. $\mathrm{P}=0.026$ when compared to mock irradiated control by student's T test. Foci were scored in 200 nuclei per sample. (c) Cells were positioned adjacent to in beam samples (approximately $35 \mathrm{~mm}$ from the centre of the beam) and were reseeded at clonal density post irradiation in parallel with directly irradiated cells. The dose at $35 \mathrm{~mm}$ from the beam centre is estimated to be $<10 \%$ of the dose at the centre of the beam.

tracks occur, can be attributed to an increased DNA lesion complexity, correlation and alteration of radical formation ${ }^{32}$. It was, however, concluded that such effects are unlikely to be observed at clinically relevant doses using high-energy protons. Conversely for antiprotons, due to the annihilation products, overlapping tracks may be expected even at low, clinically relevant, average doses. This conjecture is supported by the foci morphology and repair dynamics and confirmed by the relatively high RBE values for cell killing observed in the SOBP of the antiproton beam despite the low average LET. Taken together, these data represent strong biological evidence that it
Table 2 | Antiproton annihilation products that contribute to production of correlated DNA double strand breaks

\begin{tabular}{lccc} 
Particle & Energy $(\mathrm{MeV} / \mathrm{u})$ & Range in water $(\mu \mathrm{m})$ & $\mathrm{LET}(\mathrm{keV} / \mu \mathrm{m})$ \\
\hline Proton & 0.16 & $\sim 2$ & $\sim 70$ \\
Alpha & 1.7 & $\sim 60$ & $\sim 70$ \\
Oxygen & 1 & $\sim 17$ & $\sim 1000$ \\
\hline
\end{tabular}


Physical Dose
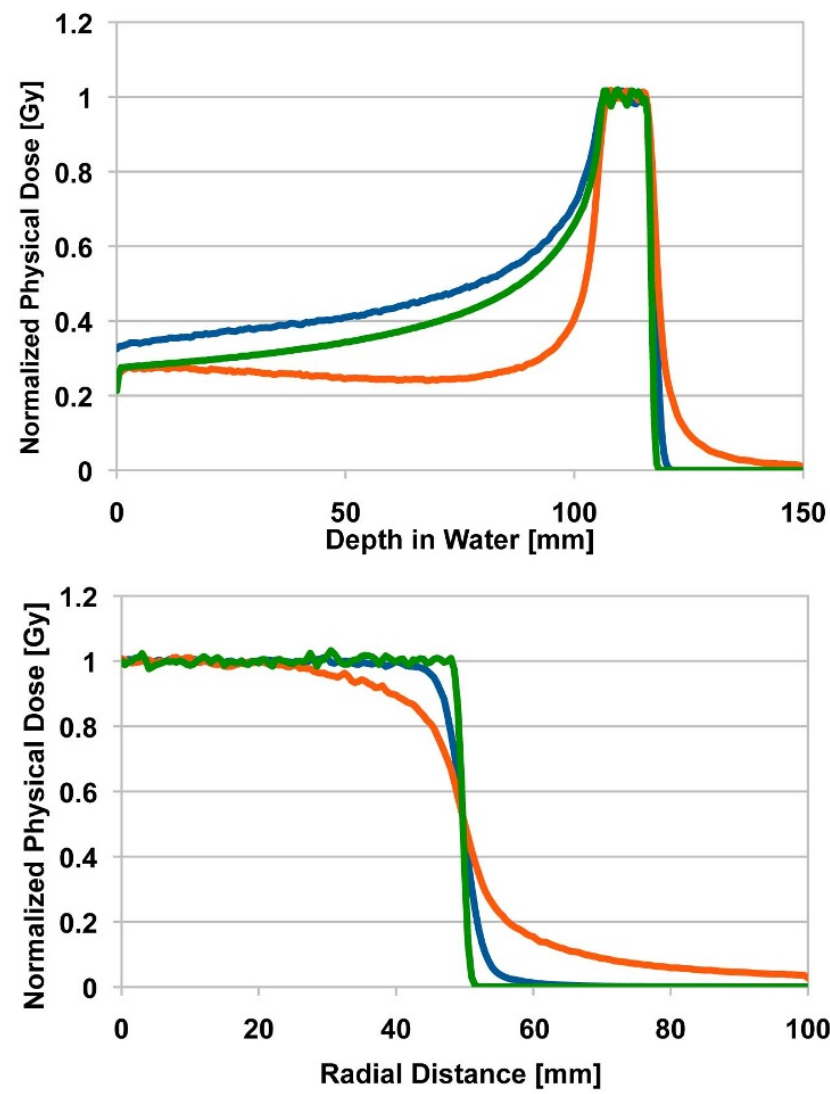

Effective Dose
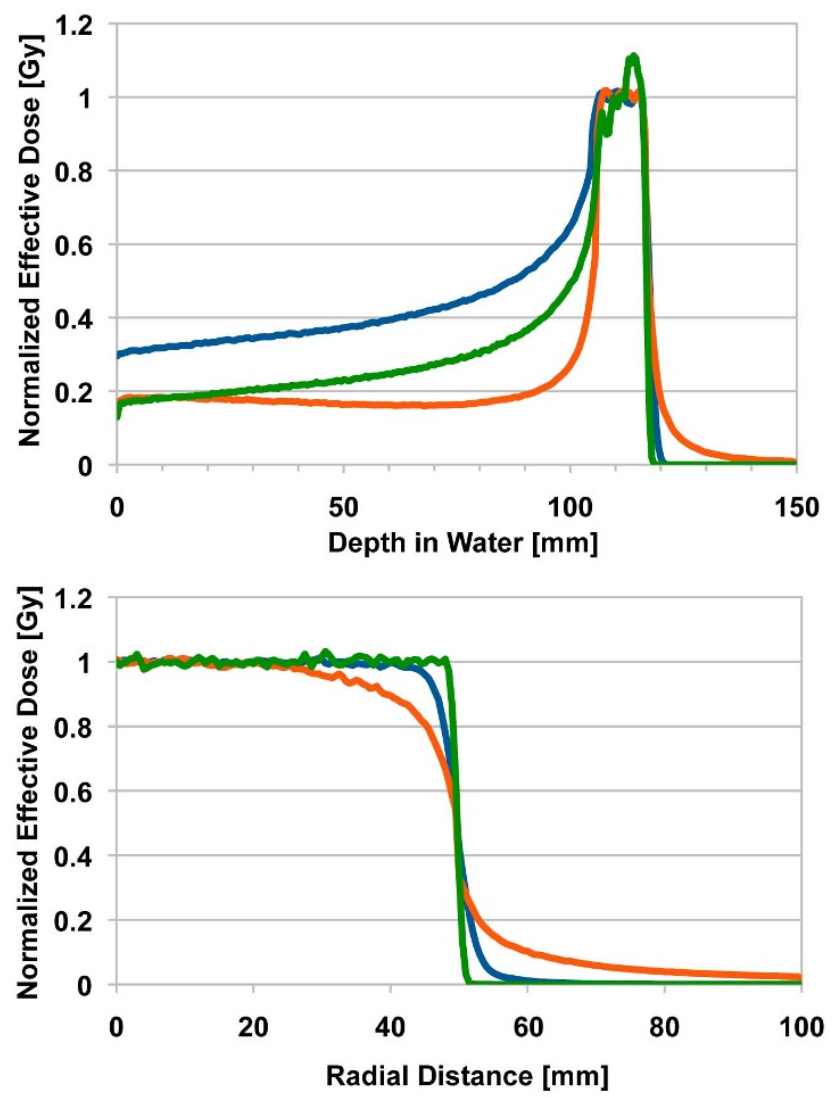

Protons - Antiprotons - Carbon lons

Figure 5 Simulated dose profiles. Depth and lateral dose profiles for simulated clinically relevant (10 mm SOBP) proton (blue line; $120 \mathrm{MeV}$ ), carbon (green line; $240 \mathrm{MeV} / \mathrm{u}$ ) and antiproton beams (red line; $120 \mathrm{MeV}$ ). Effective dose profiles obtained from the physical dose profiles and considering the $\mathrm{RBE}_{\mathrm{SOBP} / \text { plateau }}$ ratios reported in this work.

is possible to achieve an increase in biological effectiveness with low average LET radiation if the ionizations produced overlap in space and time.

The induction of large foci by antiproton SOBP observed in this study correlated with slow repair kinetics (Fig. 2) and enhanced biological effectiveness for cell killing (Fig. 3). However, it is clear from our data and from previous studies that a direct correlation between foci size and cell killing is not always evident and other factors contribute to variations in foci size ${ }^{25}$. We report an RBE for $10 \%$ survival of $1.48 \pm 0.17$ for the centre of the antiproton SOBP in AG01522 cells while for carbon ion SOBP the RBE for $10 \%$ survival was $3.88 \pm 0.06$. Additionally, the rate of repair of DNA damage induced in the antiproton SOBP was slow relative to that induced in the antiproton plateau or by protons or X-rays but was faster than carbon ion induced DNA damage. The repair rate of antiproton SOBP induced DSBs may be more comparable with that observed in the plateau region of high-energy heavy ions such as reported for oxygen ions $(14 \mathrm{keV} / \mu \mathrm{m})^{33}$. From this we conclude that annihilation induces correlated DNA lesions that are resolved slowly by the cellular DNA repair machinery due to the temporal coincidence of these insults and their spatial proximity. However, because the lesions are individually less complex than those caused by heavier charged particles, they represent a less challenging substrate for DNA repair machinery than clustered lesions caused by high LET particles such as carbon ions.

Concerning the overall antiproton SOBP RBE for survival, here we report an RBE at $10 \%$ survival of $1.48 \pm 0.17$ for the centre of the antiproton SOBP (Fig. 3). This is lower than has been reported for V79 hamster cells when a $47 \mathrm{MeV}$ antiproton beam was used ${ }^{34-36}$. This can be expected due to the higher resistance to conventional radiation of the V79 rodent cell line, compared to the human fibroblast line used in the present study. It has been seen in other studies that in general a more substantial increase in RBE is observed when the cell type in question is resistant to cell killing by low LET radiation $^{37}$. In addition, the SOBP used in our study was deeper and broader to more closely represent a clinical SOBP however this tends to dilute the dose-averaged LET $^{38}$. Cell survival data confirm the unique quality of damage induced by antiprotons with an RBE in the entrance channel statistically similar to that measured with proton and X-ray beams and an enhanced effectiveness ( $\sim 50 \%$ enhancement) in the centre of the SOBP. More importantly, it is interesting to compare the ratio of the RBE values at the SOBP and plateau for the three different particle beams. We observe no difference for protons $\left(\mathrm{RBE}_{\mathrm{SOBP}} / \mathrm{RBE}_{\text {plateau }} \sim 1\right)$ but an increase in effectiveness for antiprotons $\left(\mathrm{RBE}_{\mathrm{SOBP}} / \mathrm{RBE}_{\text {plateau }} \sim 1.5\right)$ that is, however, smaller than what was observed with carbon ions $\left(\mathrm{RBE}_{\mathrm{SOBP}} /\right.$ $\mathrm{RBE}_{\text {plateau }} \sim 1.9$ ).

For carbon ions, it is unlikely that these ratios reflect the clinical scenario. $70 \mathrm{keV} / \mu \mathrm{m}$ carbon ions may be considered to be more representative of the proximal part of the SOBP and not a true plateau that would describe the entrance channel for a clinical beam. It was not possible to create an SOBP within constraints of this study that would accurately mimic this. An RBE of 1.2 has been previously reported for AG01522 cells irradiated in the plateau of a $195 \mathrm{MeV} / \mathrm{u}$ 
carbon ion beam $(\mathrm{RBE} \sim 2.4 \text { in the peak })^{9}$ while an $\mathrm{RBE}$ of 1.33 has been reported in the same cell line following irradiation in the plateau of a $270 \mathrm{MeV} / \mathrm{u}$ carbon ion beam $(\mathrm{RBE} \sim 3.2 \text { in the peak })^{5}$. The peak to plateau ratios are therefore 2 and 2.4 respectively. These ratios suggest that the actual advantage in terms of cell killing in the target volume compared to the entrance channel is slightly higher for clinical beams than predicted from data reported here.

Possible clinical implications for the relative increase in effectiveness for antiprotons (and for protons and carbon ions) will have to be assessed against the dose profiles that can be obtained with such beams. Although more extensive and robust experimental data (including in-vivo investigations) and moreover other factors will have to be taken into account for accurate clinical evaluations, the following analysis is aimed to provide an insight on the importance of correctly estimating the biological effectiveness when dealing with ion beams. This is summarized in Figure 5. The effective dose profiles were obtained by multiplying the physical dose calculated via Monte Carlo simulations by the RBE values reported in this manuscript. Effective dose profiles for protons and carbon ions are in qualitative agreement with those reported in literature ${ }^{39}$ accounting for differences in the RBE due to the different cell line and experimental protocols/setup used. These profiles indicate that the increased $\mathrm{RBE}$ of the antiproton end-track helps to sharpen the dose distribution, as expected. However, the increased effectiveness reported here is modest and significantly higher dose levels are still expected in the distal and radial areas around the SOBP compared to protons. For the beam configurations simulated, while carbon ions exhibited the sharpest profiles, $\sim 13-15 \%$ of the antiproton target dose was delivered at $5 \mathrm{~mm}$ from the edges of the SOBP compared to less than $3 \%$ for protons. Across the whole entrance channel $(\sim 10 \mathrm{~cm})$, on the other hand, antiprotons exhibit by far the sharpest dose profiles with a factor of two reduction in entrance dose compared to protons when the RBE is considered. The total amount of normal tissue exposed and also the long term consequences for cells exposed outside the SOBP region (i.e. $\mathrm{RBE}_{\text {plateau }}$ for late cellular effects such as mutation and transformation), also need to be assessed and considered for a clinical evaluation of the use of antiproton beams. In this respect outof-field effects represent also a critical issue and further radiobiological studies are necessary in order to correctly evaluate the clinical implications of these findings.

Finally, with regards to the out-of-field effects observed in this study, no significant increase in DNA damage over the controls was observed in the out-of-field samples $1 \mathrm{~h}$ post irradiation (except for the highest dose point and the closest distance). The out-of-field dose at $3 \mathrm{~cm}$ from the beam center is expected to be less than $10 \%$ of the dose delivered to the directly exposed cells (and rapidly decreasing with distance following the $1 / \mathrm{r}^{2}$ trend). This was expected to result in a small but detectable variation of the biological response over the background of $1.6 \pm 0.08$ foci/cell.

The lack of DNA damage detected at low doses could be related to a lower effectiveness of the secondary radiation compared to the main beam in inducing DNA DSBs and the long irradiation time $(\sim 2 \mathrm{~Gy} / \mathrm{h})$ during which cellular metabolic rates are reduced (the cells were irradiated at $4{ }^{\circ} \mathrm{C}$ ) but are not completely stopped. Secondary radiation is composed of a mixture of radiation (including some high LET fragments and long range neutrons) with close spatio-temporal relation as produced by individual annihilation events. DNA damage and survival data, however, clearly demonstrated that the quality of the damage induced by the out-of-field radiation is not as severe as that caused by direct exposure to the antiproton beam and indicated an overall RBE close to 1 for the secondary particles. In the present study samples were positioned between 28 and $42 \mathrm{~mm}$ outside the antiproton beam. In a previous study using a $47 \mathrm{MeV}$ antiproton beam, DNA damage detected using the Comet assay returned to control levels in cell samples positioned just $3 \mathrm{~mm}$ distal to the Bragg peak ${ }^{34}$. Therefore, while it is accepted that annihilation particles will contribute to a dose penumbra around the SOBP, the biological effectiveness of those particles is expected to be low and their ability to cause measurable DNA damage decreases sharply with distance from the SOBP.

\section{Methods}

Cell culture. AG01522 human fibroblast cells were routinely cultured in T-175 flasks (NUNC) at $37^{\circ} \mathrm{C}$ in an atmosphere of $95 \%$ air $/ 5 \% \mathrm{CO}_{2}$. Cells were grown in filtered alpha-minimum-essential-medium (AMEM) (LONZA, BioWhittaker $\left.{ }^{\circledR}\right)$ containing foetal bovine serum $(15 \%)$, penicillin $(100 \mathrm{IU} / \mathrm{ml})$ and streptomycin $(100 \mu \mathrm{g} / \mathrm{ml})$. Cells at passage 8 were used in these experiments.

Analysis of DNA damage foci induction and repair. $3 \times 10^{5}$ cells were seeded in slide flasks (NUNC) and allowed to adhere for at least $6 \mathrm{~h}$. For irradiations the slide flasks were filled with fresh culture medium chilled to $4{ }^{\circ} \mathrm{C}$. Samples were maintained at $4^{\circ} \mathrm{C}$ during the irradiation and for up to $24 \mathrm{~h}$. Post irradiation, cold media was replaced with $3 \mathrm{~mL}$ warm media per slide flask. Cells were then incubated at $37^{\circ} \mathrm{C}$ for 1 to $24 \mathrm{~h}$, then fixed in ice-cold methanol/acetone $(1: 1)$ for $20 \mathrm{~min}$. Fixed cells were washed once in PBS then permeabilized with PBS/0.5\% Triton ${ }^{\circledR} \mathrm{X}-100$ (SigmaAldrich) for $20 \mathrm{~min}$ at $4^{\circ} \mathrm{C}$; non-specific binding was blocked with $\mathrm{PBS} / 0.2 \%$ skimmed milk $/ 5 \%$ foetal bovine serum $/ 0.1 \%$ Triton ${ }^{\circledR} \mathrm{X}-100$ for $1 \mathrm{~h}$ at $20^{\circ} \mathrm{C}$. Cells were incubated for $1 \mathrm{~h}$ at $20^{\circ} \mathrm{C}$ with anti-phospho-H2AX ( $1: 10000$ dilution, mouse monoclonal antibody, clone JBW301, Upstate Biotechnology). Cells were washed twice in PBS/0.1\% Triton ${ }^{\circledR} \mathrm{X}-100$ and then incubated for $1 \mathrm{~h}$ at $4{ }^{\circ} \mathrm{C}$ in the dark with Alexa Fluor ${ }^{\circledR} 488$ goat anti-mouse IgG cross-absorbed antibody $(1: 2000$ dilution, Molecular Probes). Cells were washed twice in PBS/0.1\% Triton ${ }^{\circledR X}-100$ and then rinsed in PBS. Slides were mounted with Vectashield mounting medium containing 4',6-diamidino-2-phenylindole (DAPI) (Vector Labs, UK). Nail varnish was used to attach cover slips to slides. Samples were scored and images acquired using a Carl ZEISS Axiovert $200 \mathrm{M}$ fluorescence microscope with CCD acquisition capability and Axiovision Rel. 4.6 software. An x63 magnification oil immersion objective was employed for scoring foci by eye. Foci were scored in 100-200 cell nuclei per sample. The scorer was blinded to the sample treatment. Unless otherwise stated all data are mean and standard error of three independent experiments. The background number of foci in sham-irradiated controls was $1.6 \pm 0.08$ foci/cell. Dose response curves were fitted using non-linear regression and linear regression analyses programs in Graph pad 4.0 software (Prism).

Foci analysis. Images of ionizing radiation-induced foci (RIF) from at least 20 cells were imported into Image $\mathrm{J}^{40}$. Intensity profiles were plotted and used to determine the full width at the maximum intensity (FWHM) for each focus. FWHM was calculated for at least 30 foci in each group analyzed.

Cellular survival measurements. $3 \times 10^{4}$ cells were seeded in $30 \mu \mathrm{L}$ of culture media in single spots ( $\sim 3-4 \mathrm{~mm}$ diameter) in sterile, dry slide flasks (NUNC). These cells were allowed to adhere for $6-8 \mathrm{~h}$ at $37^{\circ} \mathrm{C}$. Culture media was removed and cells washed with $2 \mathrm{~mL}$ warm sterile PBS before $4 \mathrm{~mL}$ of warm media was added to each slide flask for incubation at $37^{\circ} \mathrm{C}$ for a further $10 \mathrm{~h}$.

Prior to irradiation the slide flasks were filled with cold sterile culture medium. Cells were maintained at $4{ }^{\circ} \mathrm{C}$ for up to $24 \mathrm{~h}$. A time course for the duration that the cells were maintained at that temperature was carried out to confirm that this would not significantly alter the cells plating efficiency (PE). Post irradiation cells were detached by addition of $300 \mu \mathrm{L}$ of $1 \times$ Trypsin EDTA for $5 \mathrm{~min} .2 \mathrm{~mL}$ of culture medium was then added. The cell suspensions were pipetted carefully to achieve single cell suspensions. Cells were seeded into six well plates (NUNC) with densities calculated to achieve approximately 50 colonies per well. Within each experiment three wells were plated for each density and each dose point (technical replicates). Each experiment was performed three times for each radiation type. The plates were then incubated at $37^{\circ} \mathrm{C}$ in an atmosphere of $5 \% \mathrm{CO}_{2}$ and $95 \%$ humidity for 7 to 10 days or until macroscopic colonies containing at least 50 cells could be observed. Colonies were then fixed in $100 \%$ methanol and stained with crystal violet (1\% in $80 \%$ methanol).

For carbon ion and proton beam irradiations AG01522 cells were detached from the flask with trypsin EDTA $(0.5 \mathrm{w} / \mathrm{v} \%$ in sterile PBS) and the concentration of the resulting cell suspension measured using a haemocytometer. The stock suspension was diluted in fresh culture media to obtain a concentration of $\sim 5000$ cells $/ \mathrm{ml}$. Single cells were seeded in T25 flasks (NUNC) at densities calculated to give approximately 100 colonies per flask. The cells were incubated for $12-18 \mathrm{~h}$ at $37^{\circ} \mathrm{C}$ to allow single cells to attach before irradiation. $30 \mathrm{~min}$ prior to irradiation, sample flasks were removed from the incubator and filled with pre-warmed culture medium. Dose increments were delivered to achieve cellular surviving fractions of around 0.9, 0.5, 0.1 and 0.01 . Post irradiation culture medium was aspirated to leave $7 \mathrm{ml}$ per flask and flasks were subsequently incubated at $37^{\circ} \mathrm{C}$ for 10 days.

Reference X-ray survival curves that were used for proton and carbon ion $\mathrm{RBE}$ calculation were produced following this protocol. In all experiments the plating efficiency of AG01522 cells was 15-20\%.

Antiproton irradiation. Cells were suspended in a phantom containing $18.5 \%$ glycerine in water. The temperature of the liquid was controlled electronically and maintained at $4{ }^{\circ} \mathrm{C}$. Samples were positioned perpendicular to a $126 \mathrm{MeV}$ antiproton 
beam delivered by the CERN Antiproton decelerator (AD) at a dose rate of 12-17 $\mathrm{mGy} / \mathrm{min}$ in the plateau. Plateau doses ranged from 0 to $2 \mathrm{~Gy}$. Monte Carlo dosimetry simulations using the Fluka MC particle transport code were confirmed by analysis of irradiated alanine stacks and ionization chambers ${ }^{13}$. Spread out antiproton Bragg peaks were achieved by sequentially degrading the beam with PMMA degraders. The dose average LET in the plateau is $\sim 4 \mathrm{keV} / \mu \mathrm{m}$ and in the SOBP is $\sim 19 \mathrm{keV} / \mu \mathrm{m}$. The dosimetry is described in detail elsewhere ${ }^{35}$. The antiproton beam intensity is well described by a Gaussian shape in the plane perpendicular to the beam with $\sigma=4.75 \mathrm{~mm}$ with divergence of 0.5 degrees. Direct exposed samples were confined to a $\sim 4 \mathrm{~mm}$ diameter spot across which the dose and LET were uniform within 15\%. Out-of-field dose was estimated using Monte Carlo simulations and found to be $<10 \%$ of the in-field-dose at $30 \mathrm{~mm}$ from the centre of the antiproton beam. Out-of-field dose at this distance from the main beam is mainly due to secondary radiation produced by the annihilation events with less than $7 \%$ due to scattered or diverging antiprotons (TRIM simulation). Sample-beam alignment was performed using Gafchromic films and laser reference beams.

Proton and carbon ion beam irradiations. $62 \mathrm{MeV} /$ nucleon proton and carbon ion beams were produced by the super-conducting cyclotron at the CATANA facility in the INFN-LNS in Catania, Italy. Dosimetry was performed using a Markus ionization chamber (Type 34045, PTW, Freiburg, Germany). The monoenergetic beams produced were degraded by a clinical range modulator (for protons) and a ripple filter (for carbon ions) to create spread-out Bragg peaks. The average LET was $\sim 2 \mathrm{keV} / \mu \mathrm{m}$ in the entrance and $\sim 6 \mathrm{keV} / \mu \mathrm{m}$ across the SOBP for the proton beam and $\sim 70 \mathrm{keV} /$ $\mu \mathrm{m}$ in the proximal part, and $\sim 150 \mathrm{keV} / \mu \mathrm{m}$ in the distal part of the SOBP for the carbon ion beam. Samples were irradiated at a dose rate of $1-3 \mathrm{~Gy} / \mathrm{min}$ at room temperature. The beam lines and dosimetry are previously described ${ }^{37,41}$.

X-ray irradiation. Cell were irradiated using an 'XRAD225' $225 \mathrm{kV}$ X-ray cabinet fitted with a $2 \mathrm{~mm}$ copper filter (Precision X-ray Inc., CT, USA). The instrument was calibrated with a secondary standard electrometer and chamber. Doses of 0 to $8 \mathrm{~Gy}$ were delivered to cell samples with a dose rate of $0.54 \mathrm{~Gy} / \mathrm{min}$ and at a distance of $50 \mathrm{~cm}$ from the source. Control samples were mock irradiated under the same conditions.

Monte carlo simulations. Dose-depth and lateral dose curves were generated using Geant $4^{42}$ simulating energy deposited by $100 \mathrm{~mm}$ side square beams of protons, antiprotons and carbon ions in a water. Energy range 120-126 MeV was used for protons and antiprotons and 227-239 MeV/u for carbon ions in order to generate $1 \mathrm{~cm}$ SOBP. Dose-depth curves were scored in a $2 \times 2 \mathrm{~cm}^{2}$ around the beam axis and lateral dose curves were scored in a $1 \mathrm{~cm}$ deep region across the SOBP. Effective dose profiles have been obtained by assuming $\mathrm{RBE}_{\mathrm{SOBP}} / \mathrm{RBE}_{\mathrm{Entrance}}=1.1$ for protons and 1.5 for antiprotons. The SOBP regions were identified as areas with normalized physical dose $>90 \%$ and within $50 \mathrm{~mm}$ radial distance. For carbon-ion beam, the $\mathrm{RBE}$ was assumed to linearly increase with the LET in the range $20-150 \mathrm{keV} / \mu \mathrm{m}$ as suggested by a review of data available in literature ${ }^{43}$ from an initial $\mathrm{RBE}_{\text {Entrance }}=1.5$ to an $\mathrm{RBE}_{\mathrm{SOBP}}=3.9$, as determined in this work.

Statistical analysis. Sample sets for the different radiation modalities were compared by unpaired two-tailed $\mathrm{T}$ tests. It was assumed that the foci number per nucleus and focus size would be distributed normally within each sample. P values of less than 0.05 were considered to indicate that sample means were significantly different.

1. Lomax, A. J. et al. A treatment planning inter-comparison of proton and intensity modulated photon radiotherapy. Radiother. Oncol. 51, 257-271 (1999).

2. Hall, E. J. Intensity-modulated radiation therapy, protons, and the risk of second cancers. Int. J. Radiat. Oncol. Biol. Phys. 65, 1-7 (2006).

3. Yock, T. I. \& Tarbell, N. J. Technology insight: Proton beam radiotherapy for treatment in pediatric brain tumors. Nat. Clin. Pract. Oncol. 1, 97-103 (2004).

4. Fokas, E., Kraft, G., An, H. \& Engenhart-Cabillic, R. Ion beam radiobiology and cancer: time to update ourselves. Biochimica Biophysica Acta 1796, 216-229 (2009).

5. Wang, J., Li, R., Guo, C., Fournier, C. \& W, K. W. The influence of fractionation on cell survival and premature differentiation after carbon ion irradiation. J. Radiat. Res. 49, 391-398 (2008).

6. Hamada, N. et al. LET-dependent survival of irradiated normal human fibroblasts and their descendents. Radiat. Res. 166, 24-30 (2006).

7. Tsuruoka, C., Suzuki, M., Kanai, T. \& Fujitaka, K. LET and ion species dependence for cell killing in normal human skin fibroblasts. Radiat. Res. 163, 494-500 (2005).

8. Wenzl, T. \& Wilkens, J. J. Modelling of the oxygen enhancement ratio for ion beam radiation therapy. Phys. Med. Biol. 56, 3251-3268 (2011).

9. Fournier, C., Scholz, M., Weyrather, W. K., Rodemann, H. P. \& Kraft, G. Changes of fibrosis-related parameters after high- and low-LET irradiation of fibroblasts. Int. J. Radiat. Biol. 77, 713-722 (2001).

10. Bettega, D., Calzolari, P., Hessel, P., Stucchi, C. G. \& Weyrather, W. K. Neoplastic transformation induced by carbon ions. Int. J. Radiat. Oncol. Biol. Phys. 73, 861-868 (2009).

11. Gray, L. \& Kalogeropoulos, T. E. Possible Bio-Medical Applications of Antiprotons. I. In-Vivo Direct Density Measurements: Radiography. IEEE Trans. Nucl. Sci. NS-29, 1051 (1982).
12. Gray, L. \& Kalogeropoulos, T. E. Possible Biomedical Applications of Antiproton Beams - Focused Radiation Transfer. Radiat. Res. 97, 246-252 (1984).

13. Bassler, N., Holzscheiter, M. H., Jakel, O., Knudsen, H. V. \& Kovacevic, S. The antiproton depth-dose curve in water. Phys. Med. Biol. 53, 793-805 (2008).

14. Bassler, N. et al. The antiproton depth-dose curve measured with alanine detectors. Nucl. Instrum. Meth. B 266, 929-936 (2008).

15. Sullivan, A. H. A measurement of the local energy deposition by antiprotons coming to rest in tissue-like material. Phys. Med. Biol. 30, 1297-1303 (1985).

16. Bassler, N. \& Holzscheiter, M. Calculated LET spectrum from antiproton beams stopping in water. Acta Oncol. 48, 223-226 (2009).

17. Bassler, N. et al. Antiproton radiotherapy. Radiother. Oncol. 86, 14-19 (2008).

18. Sellner, S., Welsch, C. P. \& Holzscheiter, M. H. Real time imagaing of antiprotons stopping in biological targets-novel uses of solid state detectors. Radiat. Meas. 00, 1-4 (2010).

19. Kantemiris, I. et al. Real-time imaging for dose evaluation during antiproton irradiation. Phys. Med. Biol. 55, N123-131 (2010).

20. Paganetti, H., Goitein, M. \& Parodi, K. Spread-out antiproton beams deliver poor physical dose distributions for radiation therapy. Radiother. Oncol. 95, 79-86 (2010).

21. Bassler, N. et al. Comparison of optimized single and multifield irradiation plans of antiproton, proton and carbon ion beams. Radiother. Oncol. 95, 87-93 (2010).

22. Bassler, N., Holzscheiter, M. H. \& Petersen, J. B. Neutron fluence in antiproton radiotherapy, measurements and simulations. Acta Oncol. 49, 1149-1159 (2010).

23. Rogakou, E. P., Pilch, D. R., Orr, A. H., Ivanova, V. S. \& Bonner, W. M. DNA double-stranded breaks induce histone H2AX phosphorylation on serine 139. J. Biol. Chem. 273, 5858-5868 (1998).

24. Bassler, N. et al. Bubble detector measurements of a mixed radiation field from antiproton annihilation. Nucl. Instrum. Meth. B 251, 269-273 (2006).

25. Costes, S. V., Chiolo, I., Pluth, J. M., Barcellos-Hoff, M. H. \& Jakob, B. Spatiotemporal characterization of ionizing radiation induced DNA damage foci and their relation to chromatin organization. Mutat. Res. 704, 78-87 (2010).

26. Sutherland, B. M. et al. Clustered DNA damages induced in human hematopoietic cells by low doses of ionizing radiation. J. Radiat. Res. 43 Suppl, S149-152 (2002).

27. Magnander, K., Hultborn, R., Claesson, K. \& Elmroth, K. Clustered DNA damage in irradiated human diploid fibroblasts: influence of chromatin organization. Radiat. Res. 173, (2010).

28. Goodhead, D. T. \& Nikjoo, H. Track structure analysis of ultrasoft X-rays compared to high- and low-LET radiations. Int. J. Radiat. Biol. 55, 513-529 (1989).

29. Ugenskiene, R. et al. Dose response and kinetics of foci disappearance following exposure to high- and low-LET ionizing radiation. Int. J. Radiat. Biol. 85, 872-882 (2009).

30. Desai, N. et al. In vitro $\mathrm{H} 2 \mathrm{AX}$ phosphorylation and micronuclei induction in human fibroblasts across the Bragg curve of a $577 \mathrm{MeV} /$ nucleon Fe incident beam. Radiat. Meas. 41, 1209-1215 (2006).

31. Georgakilas, A. G. \& Hada, M. Formation of clustered DNA damage after highLET irradiation: A review. J. Radiat. Res. 49, 203-210 (2008).

32. Kreipl, M. S., Friedland, W. \& Paretzke, H. G. Interaction of ion tracks in spatial and temporal proximity. Radiat. Environ. Bioph. 48, 349-359 (2009).

33. Asaithamby, A. et al. Repair of HZE-particle-induced DNA double-strand breaks in normal human fibroblasts. Radiat. Res. 169, 437-446 (2008).

34. Kovacevic, S. et al. V-79 Chinese hamster cells irradiated with antiprotons, a study of peripheral damage due to medium and long range components of the annihilation radiation. Int. J. Radiat. Biol. 85, 1148-1156 (2009).

35. Holzscheiter, M. H. et al. The biological effectiveness of antiproton irradiation. Radiother. Oncol. 81, 233-242 (2006).

36. Knudsen, H. V. et al. Antiproton therapy. Nucl. Instrum. Meth. B 266, 530-534 (2008).

37. Belli, M. et al. Effectiveness of monoenergetic and spread-out Bragg peak carbonions for inactivation of various normal and tumour human cell lines. J. Radiat. Res. (Tokyo) 49, 597-607 (2008).

38. Bassler, N., Jakel, O., Sondergaard, C. S. \& Petersen, J. B. Dose- and LET-painting with particle therapy. Acta Oncol. 49, 1170-1176 (2010).

39. Elsasser, T. et al. Quantification of the relative biological effectiveness for ion beam radiotherapy: direct experimental comparison of proton and carbon ion beams and a novel approach for treatment planning. Int. J. Rad. Oncol. Biol. Phys. 78, 1177-1183 (2010).

40. Abramoff, M. D., Magelhaes, P. J. \& Ram, S. J. Image processing with Image J. Biophotonics International 11, 36-42 (2004).

41. Cirrone, G. A. P. et al. A 62-MeV Proton Beam for the Treatment of Ocular Melanoma at Laboratori Nazionali del Sud-INFN. IEEE Trans. Nucl. Sci. 51, 3568-3662 (2004).

42. Agostinelli, S. et al. Geant4-a simulation toolkit. Nucl. Instrum. Meth. A 506, 250-303 (2003).

43. Sorensen, B. S., Overgaard, J. \& Bassler, N. In vitro RBE-LET dependence for multiple particle types. Acta Oncol. 50, (2011).

\section{Acknowledgements}

The research leading to these results has received funding from the European Union Seventh Framework Programme FP7/2007-2013 under Grant Agreement n 262010 - 
ENSAR. The EC is not liable for any use that can be made on the information contained herein. JNK was funded by a Department of Education and Learning NI scholarship. Travel costs to CERN and INFN-LNS were partly supported by Engineering and Physical Sciences Research Council (EPSRC, UK) grants EP/H017844/1 (to DJT, GS and FC) and EP/ I017550/1 (to DJT, GS, FC and KIS). MHH acknowledges support by the DFG under contract WE3565-3, the NSF under grant \# CBET 0853157, and by the EU through a Marie Curie Fellowship under contract \# PIIF-GA-2009-234814. The authors wish to sincerely thank members of the CERN AD-4 team and ACE collaboration for their assistance during beam times.

\section{Author contributions}

J.N.K., G.S., F.J.C. and D.J.T. designed and conducted experiments, interpreted the data and wrote the manuscript, K.S. helped with experimental setup and reviewed and edited manuscript, D.R. conducted high resolution foci imaging and restoration deconvolution, S.J.M. carried out Geant 4 simulations, O.H. assisted with antiproton beam time experiments and provided reagents, $\mathrm{MHH}$ antiproton experimental setup, dosimetry, reviewed and edited manuscript, N.B. antiproton experimental setup, FLUKA simulations, reviewed and edited manuscript, G.A.P.C. and F.R. did the dosimetry for carbon ion and proton beams and experimental set up at INFN-LNS, K.M.P. analysed data and reviewed and edited manuscript.

\section{Additional information}

Supplementary information accompanies this paper at http://www.nature.com/ scientificreports

Competing financial interests: The authors declare no competing financial interests.

License: This work is licensed under a Creative Commons

Attribution-NonCommercial-NoDerivs 3.0 Unported License. To view a copy of this license, visit http://creativecommons.org/licenses/by-nc-nd/3.0/

How to cite this article: Kavanagh, J.N. et al. Antiproton induced DNA damage: proton like in flight, carbon-ion like near rest. Sci. Rep. 3, 1770; DOI:10.1038/srep01770 (2013). 